\title{
Conduite à tenir face à une urgence endodontique
}

\section{RÉSUMÉ}

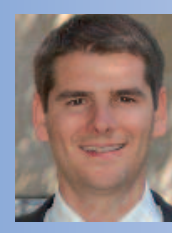

Guilhem ROMIEU

Attaché,

Ancien assistant hospitalier et universitaire

UFR Odontologie,

Université Montpellier I,

CHU de Montpellier.

7 rue du Clos,

34730 Prades-Le-Lez.

\section{Camille BERTRAND}

Assistant hospitalier et universitaire, UFR Odontologie,

Université Montpellier I,

CHU de Montpellier.

3 bis rue Galine,

34470 Pérols.

\section{Ivan PANAYOTOV}

Assistant hospitalier et universitaire, UFR Odontologie,

Université Montpellier I,

CHU de Montpellier.

UFR Odontologie,

545 avenue du Professeur Jean-Louis Viala,

34193 Montpellier Cédex.

\section{Olivier ROMIEU}

Maître de conférences,

U.F.R. Odontologie

Université Montpellier I,

CHU de Montpellier

7 rue du Clos,

34730 Prades-Le-Lez.

\section{Bernard LEVALLOIS}

Maître de conférences,

U.F.R. Odontologie,

Université Montpellier I,

CHU de Montpellier

UFR Odontologie,

545 avenue du Professeur Jean-Louis Viala,

34193 Montpellier Cédex.

La prise en charge des urgences d'origine endodontique au cabinet dentaire nécessite pour être efficace la réalisation d'un diagnostic précis et d'un acte d'urgence associé ou non à une prescription.

Pour cela, nous vous proposons des rappels concernant l'interrogatoire du patient et l'examen clinique avec la manière de réaliser les différents tests à notre disposition. Des tableaux synthétiques vous permettront de différencier les différentes pathologies en fonction des résultats des tests cliniques et d'établir le diagnostic positif et différentiel.

Une fois le diagnostic obtenu, les différentes thérapeutiques d'urgence recommandées sont également décrites.

urgence endodontique

tests cliniques

- diagnostics

thérapeutiques d'urgences
AOS 2012;259:231-244

DOI: $10.1051 /$ aos/2012304

(C) EDP Sciences 


\section{Introduction}

$>$

L'urgence dentaire, à l'exception des urgences prothétiques, correspond le plus souvent à une situation douloureuse ressentie par le patient. Il est par conséquent du devoir du praticien de recevoir ce patient afin de le soulager. Cette douleur doit être en toute circonstance prévenue, évaluée, prise en compte et traitée... (Article du Code de la santé publique L-1110-5). Elle nécessite une prise de décision et un traitement médical immédiat.

En odontostomatologie, les urgences n'engagent que très rarement le pronostic vital. Cependant, elles méritent une prise en charge rapide afin de soulager le patient et/ou d'éviter une complication.

Les urgences endodontiques en dehors des traumatismes dentaires et alvéolaires dépendent de la sévérité de l'agression, qu'elle soit d'origine bactérienne, mécanique ou chimique. De la sévérité de cette agression dépendra l'intensité de la réponse inflammatoire du tissu pulpaire et périradiculaire.

\section{Linterrogatoire}

Même dans une situation d'urgence, cette étape reste indispensable et représente le meilleur point de départ pour établir un diagnostic. II permet de recueillir des informations sur le motif de la consultation, l'état général du patient, l'anamnèse médicale et dentaire, afin de recenser les signes subjectifs de la pathologie. Une écoute attentive du patient et des questions simples à réponses fermées permettent d'orienter la recherche de la cause de la douleur.

Toutes ces informations donneront une idée assez précise du diagnostic qui devra par la suite
Les urgences endodontiques le plus souvent rencontrées sont :

- l'hyperhémie pulpaire ;

- la pulpite irréversible ;

- la parodontite apicale aiguë ;

- l'abcès apical aigu ou l'abcès phœnix ;

- la cellulite d'origine endodontique.

La prise en charge de ces urgences endodontiques passe par l'identification de la dent causale, l'établissement d'un diagnostic de la pathologie en cause, la mise en place d'une thérapeutique d'urgence adéquate accompagnée éventuellement d'une prescription médicamenteuse.

Le praticien devra donc systématiquement réaliser :

- un interrogatoire ;

- un examen clinique ;

- un diagnostic positif et différentiel ;

- un traitement d'urgence.

être confirmé ou infirmé par l'examen clinique et les tests complémentaires.

\section{Interrogatoire médical}

\section{> Sur le plan général}

Il permet de recueillir les informations sur l'état général du patient et de prendre connaissance des traitements médicaux en cours ou passés, y compris une éventuelle automédication induite par la douleur. Les éléments de ce bilan seront pris en compte dans le traitement de l'urgence. 
Le cas échéant ils contre-indiqueront tout geste invasif en urgence, nécessiteront la prescription d'une antibio-prophylaxie et éviteront des erreurs de prescription (interactions)...

\section{> Interrogatoire spécifique}

Le praticien devra poser des questions précises et compréhensibles pour recueillir les signes subjectifs le plus pertinents possible dans le but d'établir un pré-diagnostic (tableau I).

Cet interrogatoire permet de recueillir des données subjectives qui vont orienter le praticien dans une présomption de diagnostic. Celui-ci devra obligatoirement être confirmé ou infirmé par un examen clinique rigoureux avant d'entreprendre toute thérapeutique.

\begin{tabular}{|c|c|}
\hline Éléments à caractériser & Questions à poser au patient \\
\hline \multicolumn{2}{|l|}{ Douleur } \\
\hline Ancienneté de la douleur & "Depuis quand avez-vous mal ?" \\
\hline $\begin{array}{l}\text { Siège de la douleur: } \\
\text { - localisée à une dent ou diffuse } \\
\text { - secteur ou dent incriminé }\end{array}$ & $\begin{array}{l}\text { "Où avez-vous mal ?" } \\
\text { "Quelle dent vous fait mal ?" }\end{array}$ \\
\hline $\begin{array}{l}\text { Intensité de la douleur: } \\
\text { - aiguë, sourde, gêne }\end{array}$ & $\begin{array}{l}\text { "La douleur est-elle légère, modérée, forte ou intolérable ?" } \\
\text { "Sur une échelle de douleur de } 1 \text { à 10, où situeriez-vous cette douleur ?" }\end{array}$ \\
\hline $\begin{array}{l}\text { Nature de la douleur: } \\
\text { - localisée, irradiante, sourde, pulsatile }\end{array}$ & "Comment décririez-vous votre douleur?" \\
\hline $\begin{array}{l}\text { Circonstances d'apparitions : } \\
\text { - douleur spontanée ou provoquée }\end{array}$ & "Quand avez-vous mal ?" \\
\hline $\begin{array}{l}\text { Facteurs d'apparition: } \\
\text { - sucré, chaud, froid, } \\
\text { - pression, repas, décubitus }\end{array}$ & "Si elle est provoquée, par quoi ?» \\
\hline $\begin{array}{l}\text { Durée de la douleur après suppression } \\
\text { du stimulus responsable : } \\
\text { - persistante ou brève }\end{array}$ & $\begin{array}{c}\text { "Quand vous avez mal, la douleur cède-t-elle rapidement } \\
\text { ou persiste-t-elle? Combien de temps?" }\end{array}$ \\
\hline $\begin{array}{l}\text { Facteur soulageant la douleur: } \\
\text { - froid, chaud, } \\
\text { - antalgique, anti-inflammatoire }\end{array}$ & "Y a-t-il quelque chose qui vous calme?" \\
\hline \multicolumn{2}{|l|}{ Limitation fonctionnelle } \\
\hline Problème de mastication & "Pouvez-vous manger du coté douloureux ?" \\
\hline Trismus & "Avez-vous des difficultés à ouvrir la bouche?" \\
\hline \multicolumn{2}{|l|}{ Éléments complémentaires } \\
\hline Desmodontite & "Avez-vous une douleur à la mastication ?" \\
\hline Tuméfaction & "Ressentez vous un gonflement?» \\
\hline Inflammation gingivale & "Avez-vous les gencives qui saignent lorsque vous vous brossez les dents?" \\
\hline Mobilité dentaire & "Vos dents bougent-elles?» \\
\hline Foyer infectieux & "Ressentez-vous un mauvais goût ?" \\
\hline Perte de substance & "Avez-vous des dents fracturées, cassées ou trouées ?" \\
\hline Tassement alimentaire & "Avez-vous des aliments qui se coincent entre les dents?" \\
\hline
\end{tabular}




\section{Examen clinique}

Le clinicien dispose de plusieurs moyens d'examen et de toute une batterie de tests qui vont lui permettre d'établir un diagnostic positif et différentiel permettant d'identifier la pathologie. Un certain nombre d'examens et de tests sont systématiques (examen exobuccal, examen endobuccal avec inspection, palpation, percussion, sondage parodontal, mobilité), ils constituent I'examen clinique de base. En fonction de la situation, on peut être amené à compléter cet examen par d'autres tests.

\section{Examen exobuccal}

\section{> Inspection}

Rougeur, asymétrie, tuméfaction, trouble de la mobilité faciale, trouble de la phonation (fig. 1 et 10 a et b).

\section{> Palpation}

Aires ganglionnaires, contour du visage, ATM, muscles.

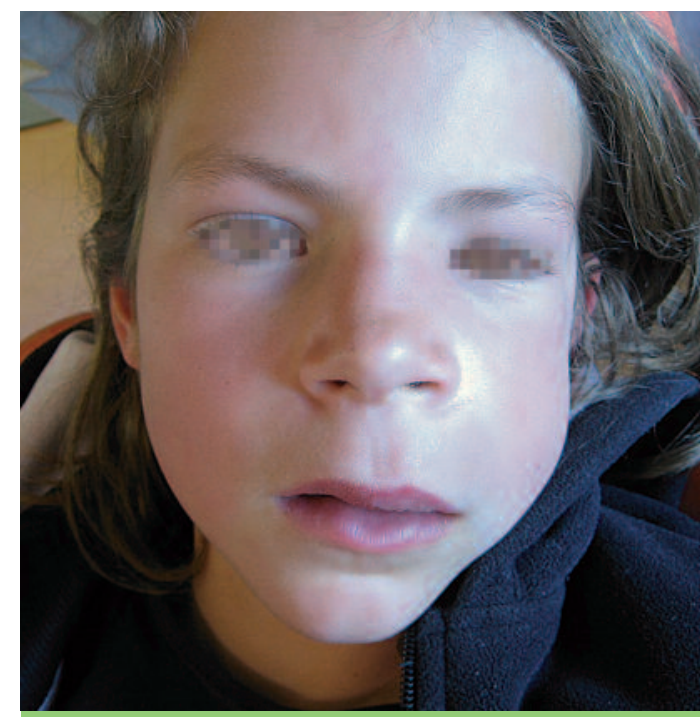

Fig. 1 Vue exobuccale d'une cellulite génienne.

\section{Examen endobuccal}

\section{> Inspection}

- Observation des tissus mous : gencives et muqueuse.

Noter la présence de tuméfaction, rougeur, ulcération, nécrose, fistule, saignement, perte de substance. Cet examen parodontal doit être systématique. II peut dans certains cas établir le diagnostic différentiel avec un abcès parodontal, une fêlure ou fracture. Une récession gingivale peut être également à l'origine d'une hypersensibilité dentinaire parfois très douloureuse.

- Observation des dents : coloration anormale, perte de substance, restaurations, prothèses.

\section{> Palpation}

Cet examen est conduit avec l'index que l'on fait glisser le long du vestibule et des procès alvéolaires au niveau des apex (fig. 2).

On recherche :

- une zone douloureuse pouvant correspondre à une lésion ou un apex extracortical ;

- une voussure en rapport avec une lésion ;

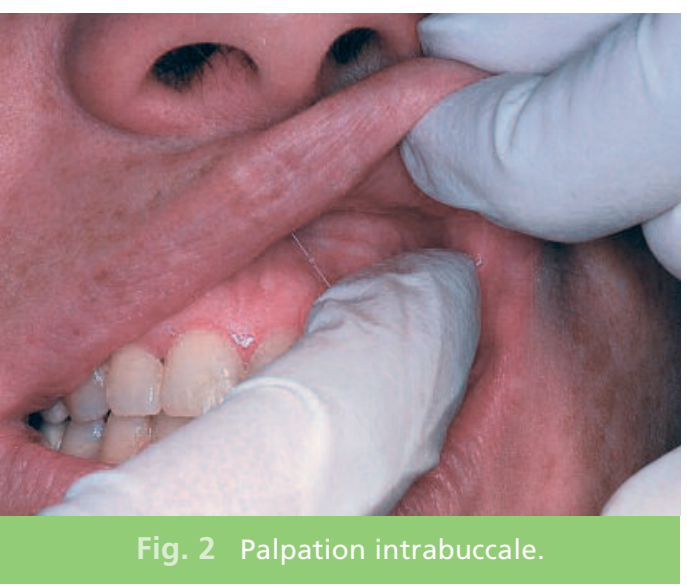




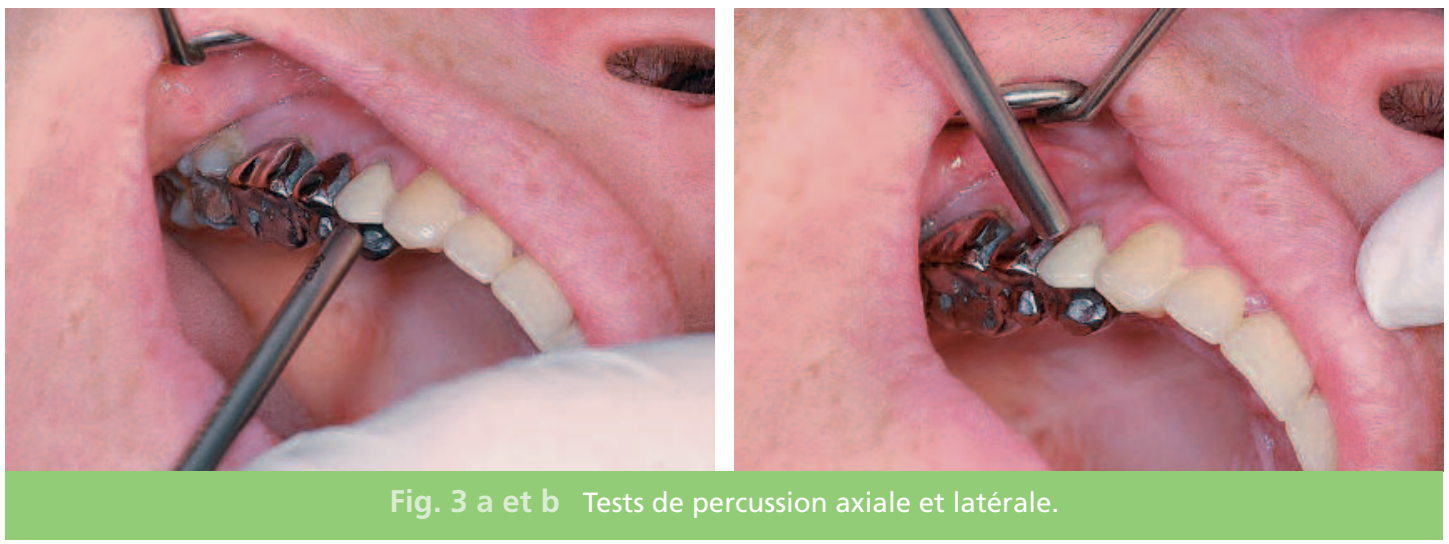

- une tuméfaction diffuse (signe du godet) ou collectée et fluctuante.

\section{> Percussion axiale et latérale}

Ce test est réalisé avec le manche d'un miroir. II peut être vertical, dans l'axe de la dent ou horizontal, perpendiculaire au grand axe de la dent (fig. 3 a et b). Il est réalisé en premier sur une dent supposée saine et à distance de la dent causale ou secteur mis en cause au cours de l'entretien. Il permet au patient d'assimiler la sensation perçue comme celle d'une sensation normale. On progresse ensuite vers la dent suspectée afin de comparer la réponse normale à celle obtenue. Une réponse douloureuse signe une inflammation desmodontale dite desmodontite. Ce test ne renseigne nullement sur l'état pulpaire de la dent. S'il est associé à la palpation des apex, un choc en retour au niveau de l'index met alors en évidence l'absence de corticale vestibulaire. Un son métallique peut être également perçu et doit nous faire suspecter une ankylose de la dent.

\section{> Sondage parodontal}

C'est un élément diagnostique indispensable pour différencier une atteinte parodontale, endodontique, combinée ou une fêlure (fig. 4). Le sondage sulculaire est réalisé avec une sonde parodontale graduée afin de rechercher une perte éventuelle du système d'attache de la dent causale. II se différencie du sondage parodontal par la pression que l'on imprime à la sonde parodontale. Celle-ci va être promenée par petits mouvements verticaux tout autour du collet de la dent suspectée. Si un sondage graduel ou progressif d'aspect en cuvette oriente le diagnostic vers un problème parodontal, un sondage profond et ponctuel signe dans la majorité des cas une fissure ou fracture. Cependant il est important de savoir faire le diagnostic différentiel avec: - une fistule desmodontale d'origine endodontique :

- un défaut embryologique de la dent tel que le sillon palatin cingulaire de l'incisive latérale maxillaire ou une perle ou une coulée d'émail au collet des molaires.

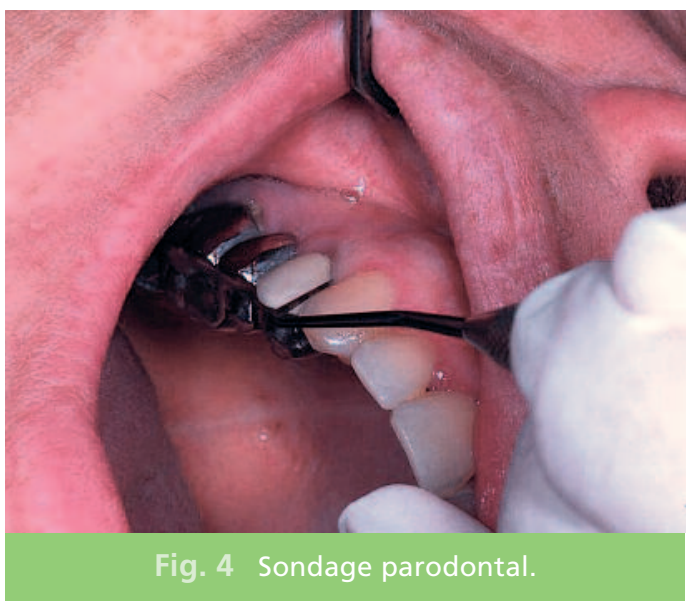


Ces fistules desmodontales se rencontrent le plus souvent au niveau des molaires mandibulaires car le desmodonte constitue une zone de moindre résistance où le drainage peut s'effectuer plus facilement. Parfois le trajet fistuleux traverse la corticale externe, soulève le périoste et s'extériorise dans le sulcus. D'un point de vue clinique, douleur et gonflement sont alors présents et cet abcès d'origine endodontique peut être confondu avec un abcès d'origine parodontale.

\section{$>$ Mobilité}

Réalisée entre deux manches d'instruments métalliques, c'est un élément qui renseigne sur l'intégrité du support de la dent.

\section{$>$ Les tests de vitalité pulpaire}

Ces tests sont indispensables pour connaître l'état de vitalité pulpaire d'une dent et confirmer le diagnostic.

Pour être fiables, ces tests doivent être effectués:

- sur des dents parfaitement sèches ;

- au niveau du tiers cervical vestibulaire des dents (en l'absence de restauration) afin d'être le plus près possible de la pulpe ;

- sur les dents adjacentes et controlatérales, afin de permettre au patient d'assimiler la sensation perçue comme celle d'une sensation normale. La dent suspectée sera testée en dernier afin que le patient puisse comparer les sensations perçues.

La dent causale devra subir les trois tests de vitalités : électrique, froid, chaud. Au moins deux de ces tests devront donner les mêmes réponses pour valider le résultat.

\section{- Test électrique}

II nécessite l'utilisation d'un " pulp tester » électrique qui transmet un courant électrique d'intensité croissante. Un conducteur type dentifrice devra être préalablement disposé sur la face vestibulaire de la dent concernée. Il est demandé au patient de se manifester dès qu'il ressent des fourmillements. Le test électrique nous renseigne uniquement sur la vitalité de la dent. II n'existe aucune corrélation entre l'état histopathologique de la pulpe et l'échelle de graduation présente sur l'appareil. La valeur numérique indiquée peut néanmoins être utile dans le cas du suivi de la vitalité pulpaire d'une dent ayant subi un traumatisme. Une augmentation importante dans le temps doit faire suspecter une nécrose en cours.

Si pour une intensité maximale le patient ne ressent aucune douleur, alors la dent testée peut être considérée comme nécrosée. L'utilisation du pulp-tester est contre-indiquée pour les patients porteurs d'un stimulateur cardiaque.

\section{- Test au froid}

Ce test peut être réalisé avec un bâtonnet de glaçon fabriqué dans des capuchons d'aiguilles d'anesthésie ou avec un spray réfrigérant tel que le dichloro-difluorométhane pulvérisé sur une boulette de coton ou sur un coton-tige (fig. 5 a). Le coton givré sera ensuite appliqué au niveau du tiers cervical vestibulaire de la dent. Une réponse exacerbée et/ou prolongée de la dent suspectée par rapport aux dents témoins est souvent le signe d'une inflammation pulpaire.

\section{- Test au chaud}

Les dents à tester sont préalablement enduites de vaseline sur la face vestibulaire afin d'éviter que la gutta-percha chauffée sur une flamme n'adhère à la dent. On utilisera un bâtonnet de gutta-percha pour les dents antérieures et un morceau de gutta-percha placé à l'extrémité d'une spatule de bouche pour les dents postérieures (fig. 5 b). Le bâtonnet ou la spatule de bouche sont portés au-dessus d'une flamme jusqu'à ce que la gutta-percha fume. Il faut prendre soin de ne pas brûler la gutta-percha. Une 


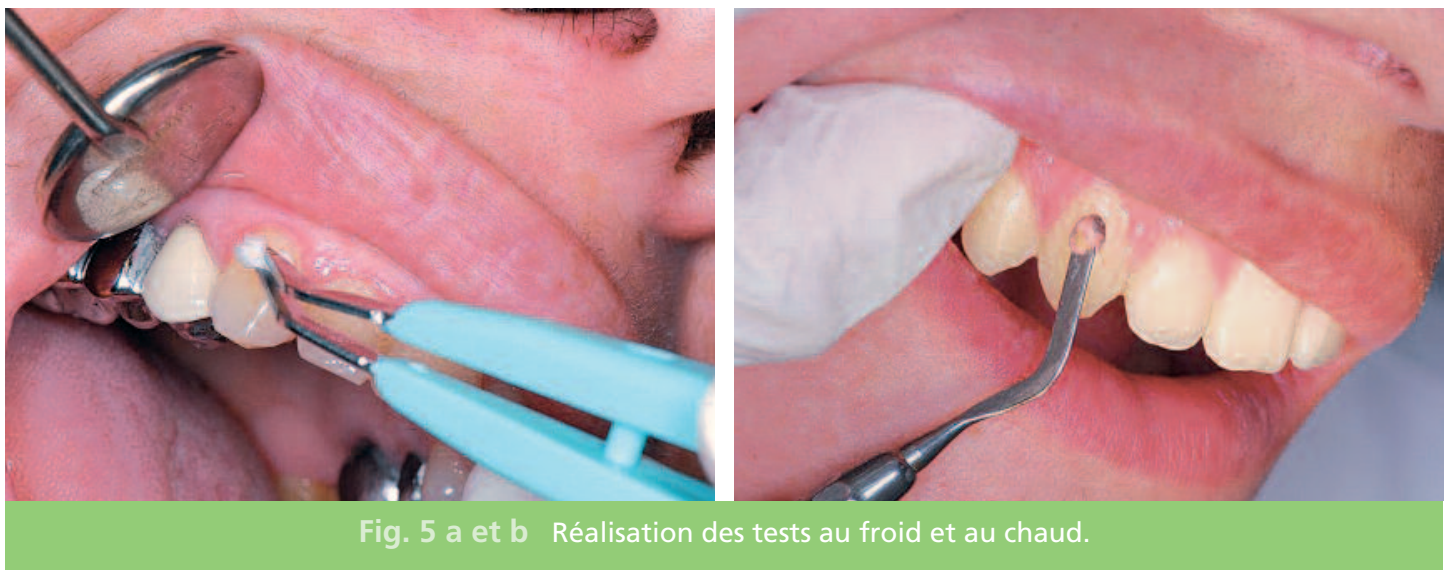

réponse positive survient généralement rapidement. Une fois cette sensation de chaleur perçue, on retire immédiatement la gutta-percha et on demande au patient de nous signaler si la douleur diminue ou augmente et au bout de combien de temps cette douleur disparaît. Il est prudent de prévoir de l'eau froide pour arrêter la douleur.

À la suite de ce test, plusieurs types de réponses peuvent être enregistrés :

- le patient ne se plaint d'aucune douleur. Si le test électrique et le test au froid sont également négatifs, la pulpe est certainement nécrosée ;

- le patient ressent une douleur, comparable aux dents adjacentes mais celle-ci cesse aussitôt après le retrait du stimulus. Si le test au froid et le test électrique sont également positifs, la dent est vitale ;

- le patient ressent une douleur plus vive que sur les dents adjacentes. Une fois le stimulus retiré, la douleur ne cesse pas mais au contraire augmente. II semble à ce stade que l'état inflammatoire de la pulpe soit très avancé, et ce, de façon irréversible.

\section{- Test de cavité}

Ce test n'est réalisé que si les autres tests ne sont pas concluants ou lorsque les tests ne sont pas réalisables (reconstitution prothétique coro- naire). On réalise sur la dent suspectée une cavité sans anesthésie afin d'évaluer la vitalité de la dent. Si la dent est vitale, les symptômes décrits par le patient au cours de l'interrogatoire sont immédiatement perçus.

Inversement, si la dent est nécrosée, on peut accéder à la chambre pulpaire sans susciter la moindre réaction douloureuse.

\section{$>$ Test de morsure}

Il peut être réalisé avec un coton-tige humidifié ou une feuille de digue enroulée autour d'une spatule à ciment et interposée entre les deux arcades (fig. 6). On demande alors au patient de mordre doucement dent par dent afin d'évaluer de manière précise les effets de la pression. En présence d'une fêlure, le patient signale instantanément la dent causale. Une douleur vive et brève est en général ressentie à l'ouverture dès que la pression est relâchée, alors que la douleur disparaît quand les dents sont serrées en occlusion. Le test de morsure doit être considéré comme un élément important du diagnostic des fêlures ou fractures.

\section{$>$ Transillumination}

Elle met en évidence une fêlure, une fracture ou une carie proximale par l'arrêt de la transmission lumineuse (fig. 7). 


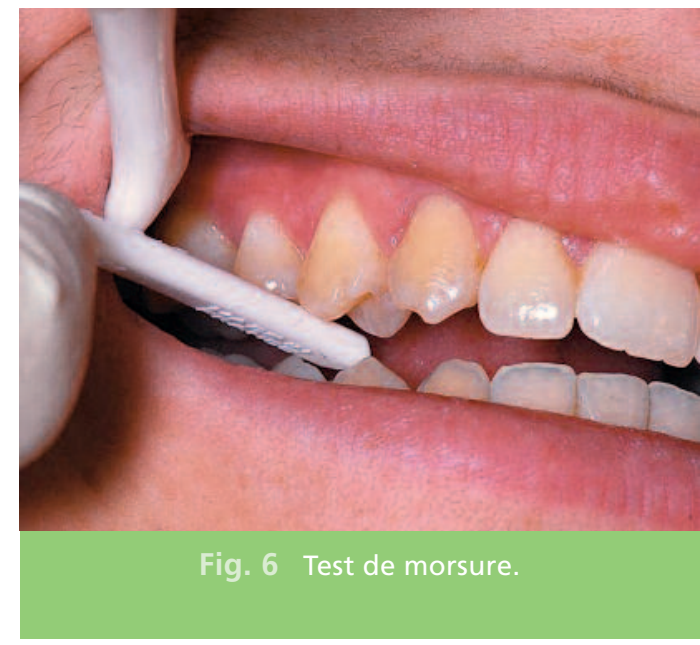

Elle peut être réalisée avec une lampe à polymériser ou mieux encore avec une fibre optique que l'on positionnera perpendiculairement à la dent examinée.

\section{> Bleu de méthylène}

Ce test est réalisé pour mettre en évidence un trait de fêlure ou de fracture.

\section{> Anesthésie sélective}

Ce test est utilisé quand on suspecte deux dents siégeant sur une arcade différente. L'anesthésie

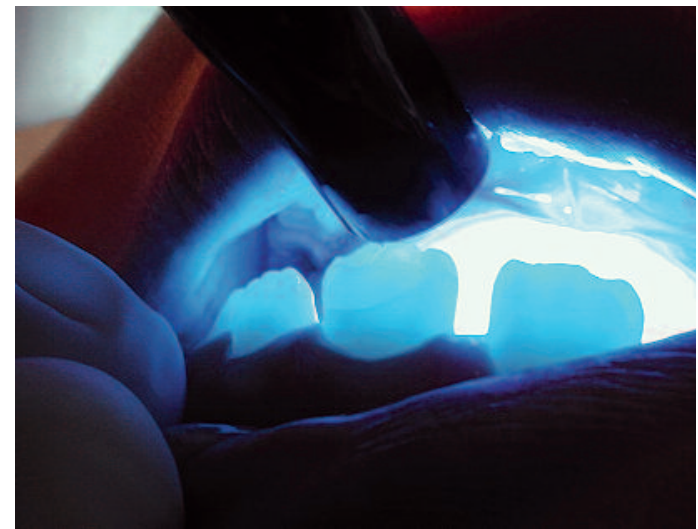

Fig. 7 Mise en évidence d'une fêlure par transillumination.

d'une des deux dents suspectées permet, en fonction de la disparition ou de la persistance des symptômes, de déterminer la dent causale.

\section{> Test du cône de gutta-percha}

Le trajet d'une fistule pourra être objectivé en y insérant un cône de gutta-percha et en réalisant un cliché radiographique qui mettra en évidence l'origine du foyer infectieux. La présence d'une fistule permet le drainage des collections suppurées et par conséquent la diminution des signes cliniques (fig. 8 a à c).
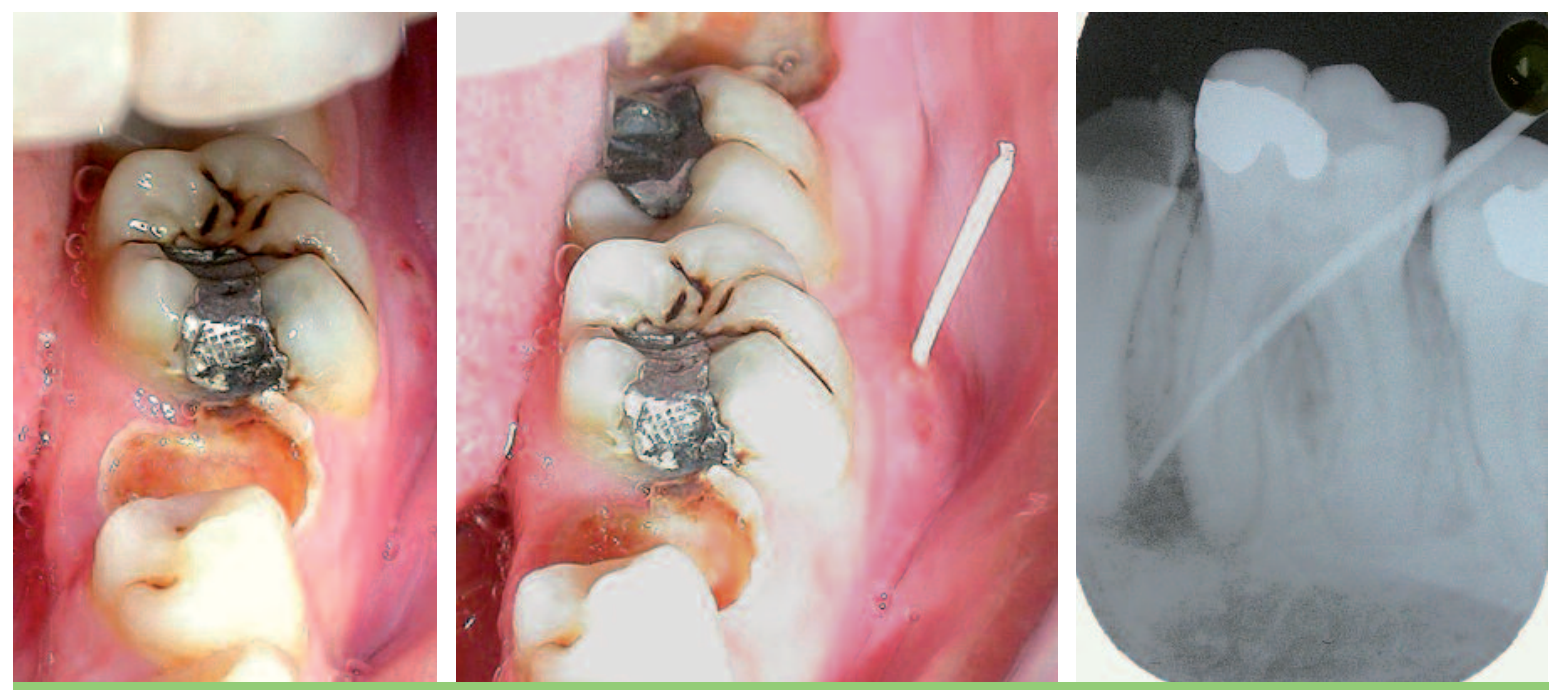

Fig. 8 a à c Vues endobuccales et radiographie avec cône de gutta-percha en place. 


\section{Examen complémentaire}

Dans le cadre de l'urgence, la radiographie est le seul examen complémentaire que l'on réalisera. Le praticien choisira le type et l'incidence les plus appropriés selon la pathologie suspectée.

- La radiographie pourra renseigner sur l'état dentaire :

- la présence d'une lésion carieuse et sa proximité avec la pulpe ;

- la proximité pulpaire d'une restauration ;

- la présence de coiffage pulpaire ;

- la présence de traitement radiculaire ;
- la présence de calcification pulpaire ;

- la présence de résorption interne ou externe ;

- la présence d'une fracture ou fêlure radiovisible.

- La radiographie pourra renseigner sur l'état parodontal :

- épaississement desmodontal ;

- continuité de la lamina dura ;

- niveau osseux, régularité de la trame osseuse ;

- trajet d'une fistule (cône de gutta) pour identifier la racine causale.

\section{Établissement du diagnostic positif et différentiel}

\section{Diagnostic positif}

$>$ Signes subjectifs

(tableau II)

> Signes objectifs

(tableau III)

\section{Diagnostic différentiel}

À l'issue de l'examen clinique, le praticien posera un diagnostic, en différentiant la pathologie du patient d'autres pathologies qui pourraient avoir les mêmes symptômes ou des symptômes proches (tableau IV).

\section{Thérapeutique d'urgence}

Les thérapeutiques d'urgence ne sont mises en œuvre que s'il n'existe pas de contre-indication sur le plan général (fig. 9 a à c). Dans le cas contraire, elles sont différées ou référées (tableau V). L'acte d'urgence représente la première étape de la thérapeutique définitive et ne doit pas compromettre les possibilités de restauration de la dent. La pose du champ opératoire intra-oral est indispensable, y compris dans le cadre de l'urgence. Dans certaines conditions, I'extraction de la dent peut constituer le traitement d'urgence de choix. 


\section{Tableau II Récapitulatif des signes subjectifs par pathologie d'origine endodontique.}

\begin{tabular}{|c|c|}
\hline Pathologies & Signes subjectifs \\
\hline $\begin{array}{l}\text { Hyperhémie pulpaire } \\
\quad= \\
\text { Pulpite réversible }\end{array}$ & $\begin{array}{l}\text { - sensibilité au sucré et au froid } \\
\text { - douleur brève qui disparaît après retrait du stimulus } \\
\text { - douleur provoquée faible à modérée } \\
\text { - localisation difficile par le patient }\end{array}$ \\
\hline Pulpite irréversible & $\begin{array}{l}\text { - symptômes récents } \\
\text { - douleur intense, pulsatile, irradiée, spontanée, prolongée rebelle aux antalgiques } \\
\text { - exacerbée par le chaud, la position allongée et l'effort physique } \\
\text { - crises douloureuses séparées par des périodes de rémission complète } \\
\text { - localisation difficile de la dent causale par le patient } \\
\text { - parfois dent douloureuse à la mastication }\end{array}$ \\
\hline Parodontite apicale aiguë & $\begin{array}{l}\text { - douleur continue, lancinante, pulsatile } \\
\text { - mastication et brossage douloureux et évités dans le secteur concerné } \\
\text { - sensation de dent « longue » } \\
\text { - localisation aisée de la dent causale par le patient }\end{array}$ \\
\hline Abcès alvéolaire aigu & $\begin{array}{l}\text { - douleurs spontanées, intenses, continues, lancinantes, nocturnes, irradiées, rebelles aux } \\
\text { antalgiques } \\
\text { - la moindre pression sur la dent exacerbe la douleur } \\
\text { - sensation de dent « longue » et mobile } \\
\text { - localisation aisée de la dent causale par le patient } \\
\text { - signes généraux (fièvre) }\end{array}$ \\
\hline $\begin{array}{c}\text { Cellulites } \\
\text { d'origine endodontique }\end{array}$ & $\begin{array}{l}\text { - tuméfaction souvent douloureuse, inflammatoire, tendue, rouge qui s'étend au travers } \\
\text { des tissus conjonctifs et des plans faciaux. } \\
\text { - la moindre pression sur la dent ou sur la tuméfaction exacerbe la douleur } \\
\text { - éventuellement signes généraux (fièvre), limitation ouverture buccale }\end{array}$ \\
\hline
\end{tabular}

\begin{tabular}{|c|c|c|c|c|c|c|c|}
\hline & 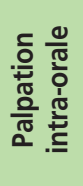 & $\frac{.}{\frac{.}{n}}$ & 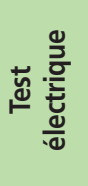 & 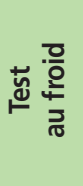 & 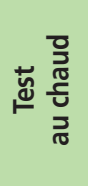 & 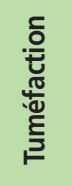 & 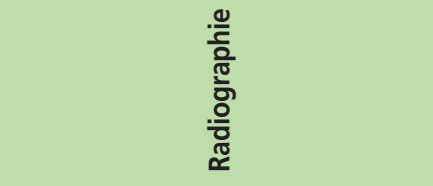 \\
\hline Pulpe saine & - & - & + & + & + & - & \\
\hline $\begin{array}{l}\text { Hyperhémie } \\
\text { pulpaire }\end{array}$ & - & - & ++ & +++ & ++ & - & $\begin{array}{c}\text { Proximité pulpaire } \\
\text { Image radio-claire coronaire } \\
\text { Restauration }\end{array}$ \\
\hline $\begin{array}{l}\text { Pulpite } \\
\text { irréversible }\end{array}$ & - & $+1-$ & ++ & + & +++ & - & $\begin{array}{c}\text { Proximité pulpaire } \\
\text { Image radio-claire coronaire } \\
\text { Restauration }\end{array}$ \\
\hline $\begin{array}{l}\text { Parodontite apicale } \\
\text { aiguë }\end{array}$ & $++/-$ & +++ & - & - & - & - & $\begin{array}{l}\text { Épaississement ligamentaire } \\
\text { à image radio-claire périradiculaire }\end{array}$ \\
\hline Abcès alvéolaire aigu & +++ & +++ & - & - & - & +++ & Image radio-claire périradiculaire \\
\hline $\begin{array}{c}\text { Cellulite } \\
\text { d'origine endodontique }\end{array}$ & +++ & +++ & - & - & - & +++ & Image radio-claire périradiculaire \\
\hline
\end{tabular}




\begin{tabular}{|c|c|c|}
\hline & Principaux diagnostics différentiels & Élément discriminant \\
\hline $\begin{array}{c}\text { Hyperhémie pulpaire } \\
= \\
\text { Pulpite réversible }\end{array}$ & $\begin{array}{l}\text { - Hyperesthésie dentinaire } \\
\text { - Sinusite }\end{array}$ & $\begin{array}{l}\text { - Récession parodontale, mylolyse, } \\
\text { historique de soins récents } \\
\text { - Situation anatomique et palpation }\end{array}$ \\
\hline Pulpite irréversible & $\begin{array}{l}\text { - Syndrome du septum } \\
\text { - Douleur neurologique/irradiée } \\
\text { - Hyperesthésie dentinaire } \\
\text { - Otite } \\
\text { - Sinusite }\end{array}$ & $\begin{array}{l}\text { - Carie proximale, compression de la papille, } \\
\text { circonstance d'apparition } \\
\text { - Absence de facteur causal dentaire identifié } \\
\text { - Récession parodontale, mylolyse, } \\
\text { historique de soins récents } \\
\text { - Siège et hypoacousie } \\
\text { - Situation anatomique et palpation }\end{array}$ \\
\hline $\begin{array}{l}\text { Parodontite apicale } \\
\text { aiguë }\end{array}$ & $\begin{array}{l}\text { - Abcès d'origine parodontale } \\
\text { - Trauma occlusal } \\
\text { - Fêlure, fracture } \\
\text { - Sinusite }\end{array}$ & $\begin{array}{l}\text { - Sondage, test de vitalité } \\
\text { - Contrôle de l'occlusion, test vitalité } \\
\text { - Sondage, test morsure, transillumination, } \\
\text { bleu de méthylène } \\
\text { - Situation anatomique et palpation }\end{array}$ \\
\hline Abcès apical aigu & $\begin{array}{l}\text { - Péricoronarite } \\
\text { - Abcès d'origine parodontale } \\
\text { - Ostéite } \\
\text { - Fusée arsenicale }\end{array}$ & $\begin{array}{l}\text { - Siège, dent en évolution } \\
\text { (dent de sagesse mandibulaire +++) } \\
\text { - Sondage }+ \text {, vitalité }+ \\
\text { - Anamnèse médicale, test de vitalité + } \\
\text { - Anamnèse dentaire }\end{array}$ \\
\hline $\begin{array}{c}\text { Cellulite } \\
\text { d'origine endodontique }\end{array}$ & $\begin{array}{l}\text { - Lithiase salivaire } \\
\text { - Emphysème sous cutané }\end{array}$ & $\begin{array}{l}\text { - Siège, circonstance d'apparition postprandiale } \\
\text { - Anamnèse dentaire, crépitement à la palpation }\end{array}$ \\
\hline
\end{tabular}
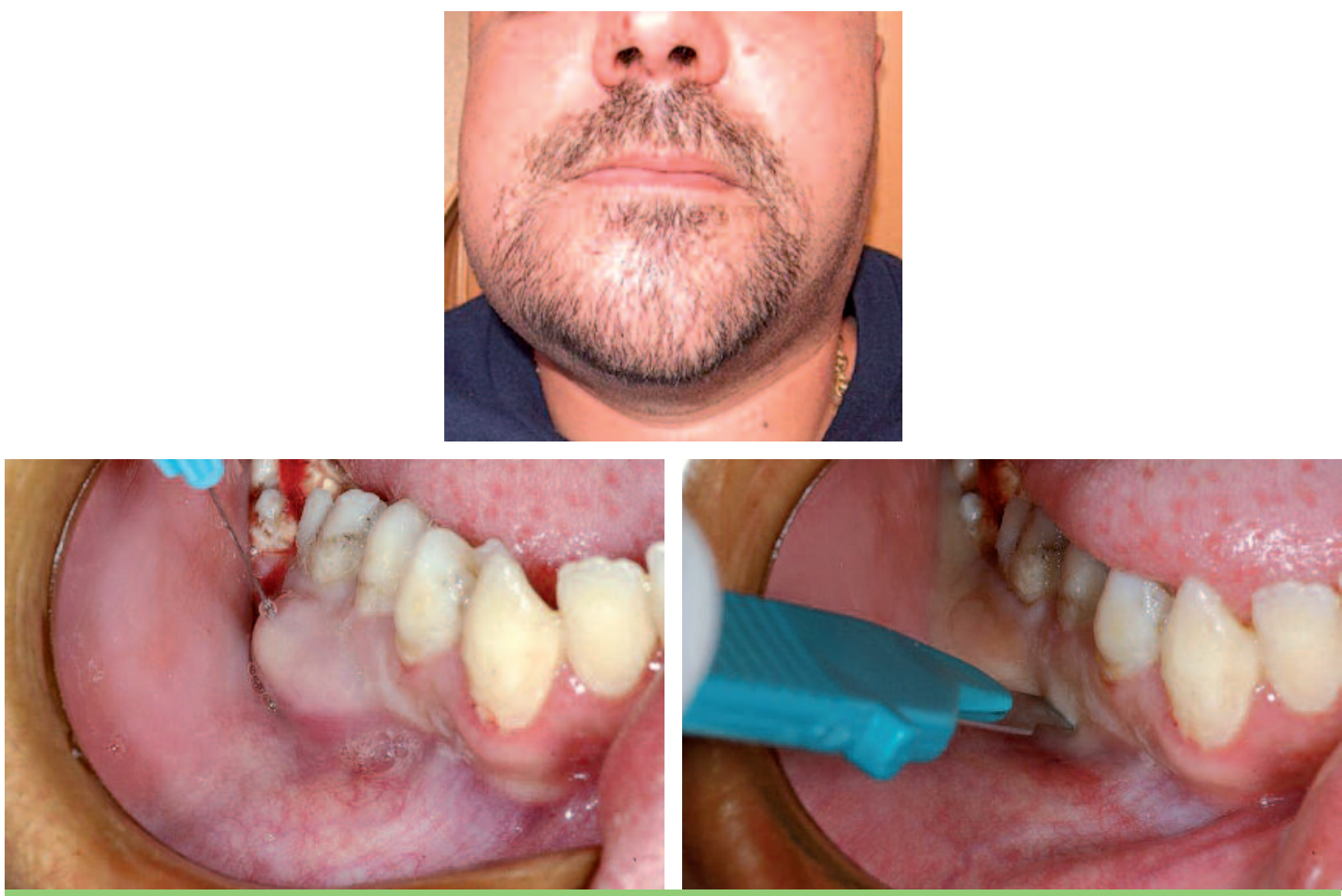

Fig. 9 a à c Vues exobuccale et endobuccale d'un abcès alvéolaire aigu et de son drainage. 
Tableau V Récapitulatif des thérapeutiques d'urgence.

\begin{tabular}{|c|c|c|}
\hline & Traitement & Prescription \\
\hline $\begin{array}{l}\text { Hyperhémie pulpaire } \\
\qquad= \\
\text { Pulpite réversible }\end{array}$ & $\begin{array}{l}\text { - Anesthésie. } \\
\text { - Curetage de la carie. } \\
\text { - Désinfection de la cavité. } \\
\text { - Obturation coronaire provisoire étanche. } \\
\text { - Coiffage pulpaire au besoin. }\end{array}$ & - Pas de prescription. \\
\hline Pulpite irréversible & $\begin{array}{l}\text { - Anesthésie. } \\
\text { - Curetage de la carie. } \\
\text { - Dépose des obturations coronaires. } \\
\text { - Biopulpectomie sur dent monoradiculée } \\
\text { avec irrigation abondante d'hypochlorite de sodium. } \\
\text { - Pulpotomie sur dents pluriradiculées } \\
\text { avec pulpectomie du canal où le saignement persiste } \\
\text { malgré une compression de } 2 \text { à } 3 \text { minutes. } \\
\text { Généralement le canal distal pour les molaires } \\
\text { mandibulaires et palatin pour les molaires maxillaires. } \\
\text { - Irrigation abondante d'hypochlorite de sodium. } \\
\text { - Obturation coronaire provisoire étanche. }\end{array}$ & $\begin{array}{l}\text { - Antibiothérapie } \\
\text { chez les patients } \\
\text { à risque infectieux. } \\
\text { - Antalgiques de niveau } 1 .\end{array}$ \\
\hline $\begin{array}{l}\text { Parodontite apicale } \\
\text { aiguë }\end{array}$ & $\begin{array}{l}\text { - Anesthésie si dent partiellement vivante. } \\
\text { - Dépose des obturations coronaires. } \\
\text { - Curetage de la carie. } \\
\text { - Mise en forme canalaire } \\
\text { + irrigation abondante d'hypochlorite de sodium. } \\
\text { - Obturation coronaire provisoire étanche. } \\
\text { - Mise-en sous occlusion éventuellement. }\end{array}$ & $\begin{array}{l}\text { - Antibiothérapie } \\
\text { chez les patients } \\
\text { à risque infectieux. } \\
\text { - Antalgiques de niveau } 1 .\end{array}$ \\
\hline Abcès apical aigu & $\begin{array}{l}\text { - Curetage de la carie. } \\
\text { - Dépose des obturations coronaires. } \\
\text { - Évacuation spontanée du pus par voie canalaire } \\
\text { après débridement canalaire et irrigation au ClONa } \\
\text { ou provoqué par la mise en place d'une lime fine } \\
\text { dans le canal et légèrement au delà du foramen. } \\
\text { La dent est laissée ouverte pendant } 24 \text { à } 48 \mathrm{~h} \text { maximum. } \\
\text { - Éventuellement mis-en sous occlusion. } \\
\text { - Si échec du drainage par voie canalaire et abcès collecté } \\
\text { et fluctuant, un drainage trans-muqueux est réalisé } \\
\text { par incision franche jusqu'au contact osseux. } \\
\text { Un drain est mise en place pour } 48 \text { h. } \\
\text { - En absence de drainage canalaire } \\
\text { et abcès non encore collecté et non fluctuant } \\
\text { laisser la dent ouverte } 24 \mathrm{~h} \text { à } 48 \mathrm{~h} \text { maximum. } \\
\text { - Dans tous les cas revoir le patient au bout de } 48 \mathrm{~h} \\
\text { avec nettoyage et désinfection du système canalaire } \\
\text { sous irrigation abondante de ClONa et fermeture de } \\
\text { la dent avec un pansement coronaire provisoire étanche. } \\
\text { Le traitement endodontique est réalisé ultérieurement } \\
\text { si possible pendant la phase d'antibiothérapie. }\end{array}$ & $\begin{array}{l}\text { - Antibiothérapie. } \\
\text { - Antalgique voire corticoïde } \\
\text { chez tous les patients. } \\
\text { - Bain de bouche chaud } \\
\text { si dent ouverte. }\end{array}$ \\
\hline $\begin{array}{c}\text { Cellulite } \\
\text { d'origine } \\
\text { endodontique }\end{array}$ & $\begin{array}{l}\text { - En absence de limitation de l'ouverture buccale, } \\
\text { la thérapeutique d'urgence sera identique } \\
\text { à celle d'un abcès apical aigu. } \\
\text { - Prise en charge hospitalière éventuelle. }\end{array}$ & $\begin{array}{l}\text { - Antibiothérapie } \\
\text { à spectre ciblé } \\
\text { sur les anaérobies. } \\
\text { - Antalgique. }\end{array}$ \\
\hline
\end{tabular}



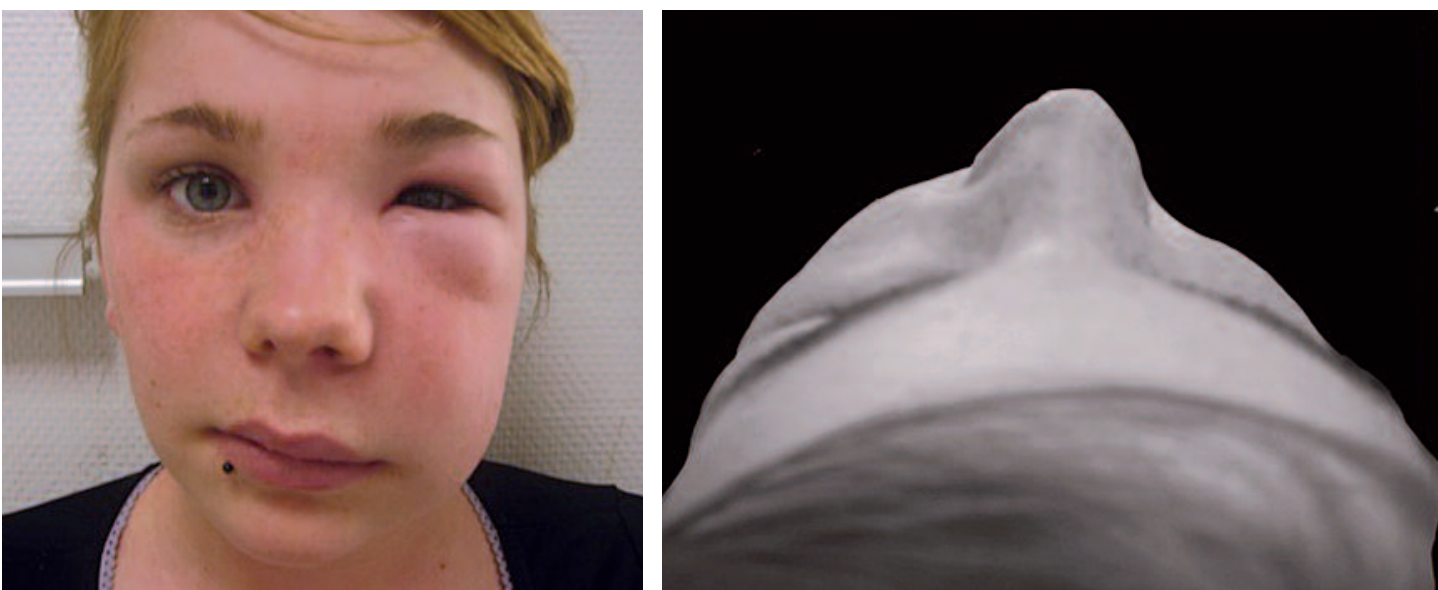

Fig. 10 a et b Vues exobuccales d'une cellulite gauche.

\section{Conclusion}

Les urgences endodontiques représentent la majorité des motifs de consultation en urgence.

Tous les patients présentant une urgence dentaire doivent être reçus rapidement, ce qui impose une organisation de l'équipe soignante et de l'agenda. Traiter l'urgence, c'est d'abord soulager le patient en atténuant la douleur mais aussi en limitant les risques de complications locales et/ou générales. Le traitement administré en urgence ne doit pas compromettre la restauration ultérieure de la dent. Les contraintes de temps et le stress du patient ne doivent pas détourner le praticien des bonnes pratiques cliniques.
Un diagnostic précis et une connaissance claire et précise des conduites à tenir permettent au praticien de pratiquer les gestes appropriés et rendent I'acte thérapeutique sûr, efficace et rapide car une infection apicale peut avoir des conséquences gravissimes et constituer une urgence vitale.

Il est nécessaire d'informer le patient que la thérapeutique mise en œuvre dans le cadre de l'urgence n'est bien souvent que symptomatique et que des soins définitifs devront être entrepris. Les patients que l'on réussit à soulager efficacement sont généralement très reconnaissants envers le praticien qui les a reçus rapidement.

\section{Ouvrages à consulter}

- Lasfargues JJ. Les traitements endodontiques d'urgence. $1^{\text {re }}$ partie Rev Fr Endod 1984 ;3(4):17-29.
- Lasfargues JJ. Les traitements endodontiques d'urgence. $2^{\mathrm{e}}$ partie. Rev Fr Endod 1985;4(1):43-63.
- Cohen AG, Hartmann A, Machtou P. Le traitement des urgences endodontiques.

Réal Clin 1992;3(1):139-51. 
- Pertot WJ, Simon S. Le traitement endodontique. Paris : Quintessence International, 2004.

- Simon S. Endodontie. Vol. 1 : traitements. Paris : Éditions CdP, 2008.

- Hess JC, Medioni E, Vene G. Pathologie endodontique. Ensemble pulpo-dentinaire. Clinique dentinaire La dentine découverte. Paris : Encycl Méd Chir (Elsevier Masson SAS), Odontologie, 1989;23-020-A-10.

- Collège national des enseignants en odontologie conservatrice et endodontie. Dictionnaire francophone des termes d'odontologie conservatrice endodontie \& odontologie restauratrice. Paris : SNPMD, 2004.

- Collaboration canadienne pour établir des normes cliniques en dentisterie. Emergency Management of an Acute Apical Abscess in Adults, 2003.

Emergency Management of an Acute Apical Periodontitis in Adults, 2002. http:/www.cccd.ca/

- Dubernard C, Bellanger S, Chambon G, Léon $\mathrm{H}$, Torres JH Lozza J.

Cellulite d'origine dentaire engageant le pronostic vital : à propos d'un cas. Med Buccale Chir Buccale 2009;15(3):119-26.
- Jafarzadeh H, Abott PV. Review of pulpsensibility tests. Part I: General information and thermal tests. Int Endod J 2010;43(9):738-62.

- Jafarzadeh H, Abott PV. Review of pulp sensibility tests.

Part II: Electric pulp tests and test cavities. Int Endod J 2010;43(11):945-58.

- Berman LH, Hartwell GR. Diagnosis. In: Cohen S, Hargreaves K.(eds). Pathways of the Pulp. $9^{\text {th }}$ edition. St Louis : CV Mosby Company, 2006:2-39.

- Toledo-Arenas R., Descroix V. Urgences odontologiques. Paris : Elsevier/Masson, Coll. Pratique dentaire, 2010.

\section{SUMMARY}

\section{What to do in front of \\ an endodontic emergency}

Guilhem ROMIEU,

Camille BERTRAND,

Ivan PANAYOTOV,

Olivier ROMIEU,

Bernard LEVALLOIS
The management of endodontic emergencies (from dental root canal) require accurate diagnosis and emergency procedure with or without drug delivery. For this, we offer reminders for patient interview and clinical examination. Various clinical tests are describe. Summary tables based on anamnesis and clinical tests helps you for positive and differential diagnosis. Recommended emergency treatments and drug delivery are also given in this article. 